\title{
Effect of calcipotriol plus betamethasone dipropionate gel phonophoresis on psoriasis: a single-blind randomized controlled trial Ereny S. Wahba
}

Department of Physical Therapy for Surgery, Faculty of Physical Therapy, Cairo University, Giza, Egypt

Correspondence to Ereny S. Wahba, PhD, Faculty of Physical Therapy, Cairo University, 7 Ahmed Alzayate Street, Been Úlsarayat, Giza 12111, Egypt. Tel: +20 100403 7190; fax: 0020237617692 ; e-mail: ereny_sobhy2002@yahoo.com

Received 14 October 2018 Accepted 14 March 2019

Bulletin of Faculty of Physical Therapy 2019, 24:57-65

\begin{abstract}
Background and psychological, social, and physical functioning.

Objective plaque psoriasis.

Patients and methods before treatment and after 8 weeks of the treatment.

Results group compared with that of control group $(P<0.0001)$.

Conclusion therapeutic procedure in the treatment of plaque psoriasis.

\author{
Keywords: \\ betamethasone diproprionate, calcipotriol, phonophoresis, psoriasis \\ Bulletin of Faculty of Physical Therapy 24:57-65 \\ (C) 2019 Bulletin of Faculty of Physical Therapy \\ $1110-6611$
}

Psoriasis is a common, immune-mediated, inflammatory disease that can affect all aspects of a person's life including esthetic appearance, relationships with others,

This study was conducted to investigate the therapeutic benefits of calcipotriol plus betamethasone dipropionate gel (Dovobet gel) phonophoresis in the treatment of

Forty patients with a moderate degree of psoriasis (localized psoriasis) were included, and their age ranged from 25 to 45 years. Patients were randomly assigned into two groups: the study group underwent calcipotriol plus betamethasone dipropionate gel phonophoresis and the control group underwent calcipotriol plus betamethasone dipropionate gel. All treatment interventions were applied at a frequency of three sessions per week for 8 weeks. Outcome measures were performed through ultrasound imaging system that was used to measure the thickness of the skin. The assessment was done

There was a significant decrease in the skin thickness after treatment in the study

Calcipotriol plus betamethasone diproprionate phonophoresis is a useful

\section{Introduction}

Psoriasis is a common, immune-mediated, inflammatory disease especially related to the skin and joints, affecting $1-3 \%$ of the overall population [1]. Psoriasis represents a complex disorder within which genetic factors account for $\sim 70 \%$ of disease susceptibility, whereas environmental elements account for the last 30\% [2]. These findings suggest that heritability is not the simplest motive of psoriasis, and different environmental elements along with skin microbiota might also make a contribution to the susceptibility to this immunoinflammatory disorder. The estimated prevalence of psoriasis ranged from 0.51 to $11.43 \%$ in the adult population [3]. Aside from negatively affecting the quality of life, patients with psoriasis may also have an increased risk of comorbid diseases, which include diabetes mellitus, Crohn's disease, most cancers, despair, nonalcoholic fatty liver disease, metabolic syndrome, and cardiovascular illnesses [4].

Psoriasis is described by an overstated expansion of keratinocytes auxiliary to an activated immune system. The occurrence is most elevated at the age of 20-39 years in males and 40-59 years in females, with an equivalent male-to-female representation [5]. Psoriasis vulgaris or plaque psoriasis represents right around $90 \%$ of the dermatological presentation of the disease; however, a few different structures including guttate, reverse, erythrodermic, pustular, and palmoplantar psoriasis may happen and nail inclusion [6]. Topical treatment remains the backbone in the treatment of psoriasis, with more than $80 \%$ of patients treating their psoriasis with topical application alone [7]. Current rules for the mainline treatment of mild-to-moderate psoriasis suggest the topical utilization of vitamin D analogues and corticosteroids [8].

National Institute for Health and Care Excellence suggests estimating the degree of psoriasis by estimating body surface area (BSA). Seriousness is

This is an open access journal, and articles are distributed under the terms of the Creative Commons Attribution-NonCommercial-ShareAlike 4.0 License, which allows others to remix, tweak, and build upon the work non-commercially, as long as appropriate credit is given and the new creations are licensed under the identical terms. 
outlined by what proportion of the body extent is affected: mild psoriasis: less than $5 \%$ of BSA, moderate psoriasis: $5-10 \%$ of BSA, and severe psoriasis: more than $10 \%$ of BSA (note: $1 \%$ of BSA is close to adequate the palm of the patient's hand, barring fingers) [9].

Security concerns regularly associated with these monotherapies incorporate skin irritation and hypercalcemia for vitamin D analogues, skin atrophy, and adrenal suppression with semipermanent use for corticosteroids. As such, a fixed combination of a topical preparation containing calcipotriol "vitamin D analogue" and betamethasone dipropionate "corticosteroid" appeared to be very effective versus the individual components for the treatment of psoriasis [10].

The restorative advantage is achieved with the fixed combination, advancing greater anti-inflammatory and antiproliferative effects than for either active ingredient alone, combined with a faster reaction and possibly fewer safety concerns [11] compared with monotherapies. This is because of the steroid component of the fixed combination gel depressing the rate of skin irritation sometimes experienced with the utilization of vitamin D analogues, and calcipotriol possibly decreasing steroid-prompted skin atrophy [12].

The combination of calcipotriol $50 \mathrm{mg} / \mathrm{g}$ and betamethasone diproprionate $0.5 \mathrm{mg} / \mathrm{g}$ gel (Dovobet gel) has been created for the treatment of psoriasis. A lipophilic, alcohol-free gel formulation of the fixed combination preparation (Dovobet gel) was created to offer an insignificant effect on the patient's daily schedule and along these lines advance patient adherence [13]. A more cosmetically acceptable and simple-to-utilize treatment in the form of a fixedcombination lipophilic, alcohol-free gel has demonstrated favourable patient-related benefits in adults and may have increased acceptability in adolescents with psoriasis [14].

Ultrasound phonophoresis is a strategy for enhancing the skin permeation of medications; the usage of ultrasound acts as a physical power. The combination of ultrasound therapy together with topical medication therapy increases restorative drug concentrations at selected sites within the skin. In this method, the drug is joined with a coupling agent, ordinarily a gel [15].

Utilization of low-frequency ultrasound $(20-100 \mathrm{kHz})$ upgrades skin permeability more strongly than high- frequency ultrasound $(1-16 \mathrm{MHz})$. The system in regards to transdermal skin permeation includes a breakdown of the stratum corneum lipids, in this manner enabling the medication to pass by means of the skin. A comparative decrease in skin opposition by the effect of ultrasound waves as cavitation, microstreaming and heat generation [16]. Phonophoresis via ultrasound has various points of interest in that there is a low risk of burning the skin, and it is unnecessary to ionize the drug.

Effectively, medicines contained within or under the ultrasound gel are pushed by the sound waves of the ultrasound and driven far beneath the skin. Phonophoretically administered medications can penetrate the body to a much deeper level than those massaged by hand over the surface of the skin [17]. Phonophoresis offers the potential advantage of delivering a pharmacologic agent in a relatively safe, painless, and easy manner to structures that lie somewhat deep within the body [18]. So phonophoresis is a type of combination therapy that implicates both the therapeutic effects of ultrasound and enhancement the drug therapy efficacy in the treatment simultaneously, making this combination more successful than every treatment alone [19].

The requirement for this investigation has been produced in light of the fact that psoriasis has greater effect on the psychosocial aspects of the patients' lives. There are troubles in the work environment, socialization with relatives and friends, prohibition from open offices, and landing a position. Additionally, suicide was one of the real issues that were represented by these patients [4]. There are lack of data and knowledge in the physical therapy field about the efficacy of calcipotriol plus betamethasone dipropionate gel phonophoresis as a physical therapy modality in the treatment of psoriasis. The need for a new effective and localized treatment for psoriasis has become greatly important for both patient and community. The main principle of phonophoresis is to enhance drug delivery in a wide variety of cutaneous conditions. Phonophoresis of calcipotriol plus betamethasone dipropionate gel for psoriasis may represent a viable option for treatment.

Previous studies have focused only on the efficacy of calcipotriol plus betamethasone dipropionate gel, but no study has been conducted to combine this different method with usage of ultrasound waves to improve the absorption of those medications by the skin $[10,20-25]$. 
So the purpose of this study was designed to investigate the therapeutic benefits of calcipotriol plus betamethasone dipropionate gel (Dovobet gel) phonophoresis in the treatment of psoriasis.

\section{Patients and methods \\ Study design}

The study was designed as a prospective, randomized, single-blind, and pretest-post-test trail. Ethical approval was obtained from the institutional review board at the Faculty of Physical Therapy, Cairo University, before study commencement, with number P.T.REC/012/ 002118. The study has followed the Guidelines of the Declaration of Helsinki on the conduct of human research. The study was conducted between January 2018 and September 2018.

\section{Participants}

A convenient sample of 40 (20 male and 20 female) patients was recruited from the Dermatological out Patients Clinic at Faculty of Medicine, Cairo University, to be treated in the Outpatient Clinic, Faculty of Physical Therapy, Cairo University. They were enrolled and assessed for their eligibility to participate in the study. To be included in the study, patients had plaque-type psoriasis with a moderate degree of psoriasis (localized psoriasis), and their age ranged from 25 to 45 years.

Patients were excluded from the study if they had any other illness that may affect the results such as diabetes, circulatory disorders, peripheral vascular diseases, and acute viral diseases. Moreover, pregnant women; patients with any contraindication for calcipotriol plus betamethasone dipropionate gel (Dovobet; LEO Pharma, Ballerup, Denmark) such as erythrodermic, exfoliative, pustular psoriasis, fungal or bacterial skin infections, skin manifestations in relation to tuberculosis, perioral dermatitis, atrophic skin, striae atrophicae, fragility of skin veins, ichthyosis, acne vulgaris, acne rosacea, rosacea, ulcers and wounds; patients with any contraindication for an ultrasound (e.g. acute infection or malignancy in the area to be treated); patients who received biological therapies with a possible effect on psoriasis vulgaris within 1-6 months before randomization, and those who received other systemic antipsoriatic therapies and psoralen+UVA therapy within 4 weeks before randomization were also excluded.

\section{Sample size}

Using $G$ power (Heinrich-Hine University of Dusseldrof) program, a preliminary power analysis [power $(1 \alpha$ error $P)=0.85, \alpha=0.01$, effect size $=0.5$ ] determined a sample size of 40 for this study. This effect size was chosen because it yielded a realistic sample size [26].

\section{Randomization}

Informed consent was obtained from each participant after explaining the nature, purpose, and benefits of the study, informing them of their right to refuse or withdraw at any time, and informing about the confidentiality of any obtained information. Anonymity was assured through coding of all data. Participants with chronic plaque psoriasis were randomly assigned into two equal groups (study group and control group) by a blinded and an independent research assistant who opened sealed envelopes that contained a computer-generated randomization card. No patients dropped out of the study after randomization.

\section{Unrestricted (simple) randomization}

Unrestricted randomization was done as follows: combine the 20 sealed study group envelopes with the 20 sealed control group envelopes and shuffle as you would a deck of cards. Once you are satisfied that the deck of envelopes is shuffled thoroughly, with a firm hand, mark a unique number on the front of each envelope sequentially from 1 to 40 , with a pen. The carbon paper inside the envelope will transfer this number to the allocation paper inside. Place these envelopes into the plastic container, in numerical order, ready for use [27].

The patients were randomly assigned to the study group (20 patients, with 11 female and nine male) or the control group (20 patients, with 10 female and 10 male) by another independent therapist who took a sealed opaque envelope from a box following a numerical sequence; the envelope contained a letter indicating whether the patient would be allocated to the study group or the control group. Both groups were compared regarding patient demographics including age and sex.

\section{Instrumentation}

The following instruments were used:

\section{Ultrasound unit}

Sonopulse 590 Ultrasound Device: Nonius, sonopuls 590, S.NO.03-202 type 14663.900 is a therapeutic ultrasound device manufactured by Enraf Holland (Birmingham, West Midlands, UK).

\section{Ultrasonography unit}

Voluson 730Expert/Pro V. 4.0.x GE Medical Systems Kretz Ultrasound was obtained from General Electric 
Company Healthcare, which is an American multinational conglomerate incorporated in New York and headquartered in Chicago, Illinois. The Company was incorporated in 1994 and operates in more than 100 countries. GE Healthcare operates as a subsidiary of General Electric [28].

\section{Procedure}

The procedure of this study was divided into two main parts: part one (assessment procedure) and part two (treatment procedure).

\section{Assessment procedure}

Primary examination was done to every patient to be sure that there were no any contraindications to conduct the study.

The affected area was visualized to ensure that there were no contraindications before application.

\section{Assessment Equipment}

The measuring equipment was done by:

\section{Ultrasonography}

An ultrasound imaging system was used to measure the thickness of the skin at the affected area in relation to a defined reference point before and after the treatment for all patients in both groups.

\section{Measurements were carried out at E1 Mataria Teaching} Hospital (Department of Radiology).

The ultrasonographic image of psoriatic plaque is characterized by a varying increase in the width of the entry echo sign. Under this echo, a hypoechogenic band related to inflammatory and vascular changes is detectable. This band is more evident in the acute stage. The dermis appears thicker and nonhomogeneous. On comparing ultrasonic images with histological examination, three zones can be detected: A zone that represents the thickened stratum corneum, B zone that represents the elongated rete ridges and edematous papillary dermis, and $\mathrm{C}$ zone that represent the reticular dermis. The thickness of $\mathrm{B}$ zone of psoriatic plaque shows a significant correlation with the Psoriasis Area Severity Index (PASI) score [29].

The instrument combines a scanning transducer and a computer in a single instrument and can be used at the patient bedside, producing high-resolution images of human tissue. Ultrasonography was used to evaluate the thickness of the skin at the affected area in relation to a fixed point. This point was determined in relation to a certain landmark (e.g. the landmark used for thigh was greater trochanter, for leg was patella, for foot was medial and lateral malleolus, for the arm was acromion, for the forearm was olecranon, for the hand was radial or the ulnar styloid process, and for the back was spinous processes).

The $40-\mathrm{MHz}$ high-frequency ultrasound machine, with its higher resolution, has enabled us to determine the size of the bands in a psoriatic plaque more precisely than we were previously able to do with the lower-frequency systems [30].

The device was used to measure skin thickness before treatment and after 8 weeks of the study (posttreatment) for every patient in both groups.

\section{Treatment procedure}

Participants were randomly assigned to the following:

\section{Study group (Dovobet gel phonophoresis)}

Patients received calcipotriol/betamethasone dipropionate gel phonophoresis (Dovobet gel; LEO Pharma A/S). Therapist applied Dovobet gel to the affected area with the applicator and rubbed it in gently until the area affected by moderate degree psoriasis vulgaris was covered by a thin layer of gel, then the ultrasound was implemented with the following parameters: continuous mode at $100 \mathrm{~Hz}$, duty cycle of $10 \%$, frequency of $1 \mathrm{MHz}$, intensity of $1.5 \mathrm{~W} / \mathrm{cm}^{2}$ three sessions per week for eight weeks and an effective irradiation area of $1.0 \mathrm{~cm}^{2}$. Ultrasound was applied for 5 min, using the method of direct coupling and oscillatory rubbing movements [31].

\section{Control group (Dovobet gel only)}

Patients received calcipotriol/betamethasone dipropionate gel (Dovobet gel). Dovobet gel was applied to the affected area and was rubbed gently until the area affected by moderate degree psoriasis vulgaris was covered by a thin layer of gel. It was applied once per day, for 8 weeks.

\section{Statistical analysis}

All statistical analysis was conducted through the statistical package for the social studies (SPSS) version 19 for Windows (IBM SPSS, Chicago, Illinois, USA). Descriptive statistics and unpaired $t$ test were conducted for comparison of patient characteristics between both groups. $\chi^{2}$-Test was used for comparison of sex distribution between groups. Normal distribution of data was checked using the Shapiro-Wilk test. Unpaired $t$-test was conducted to compare the mean values of skin 


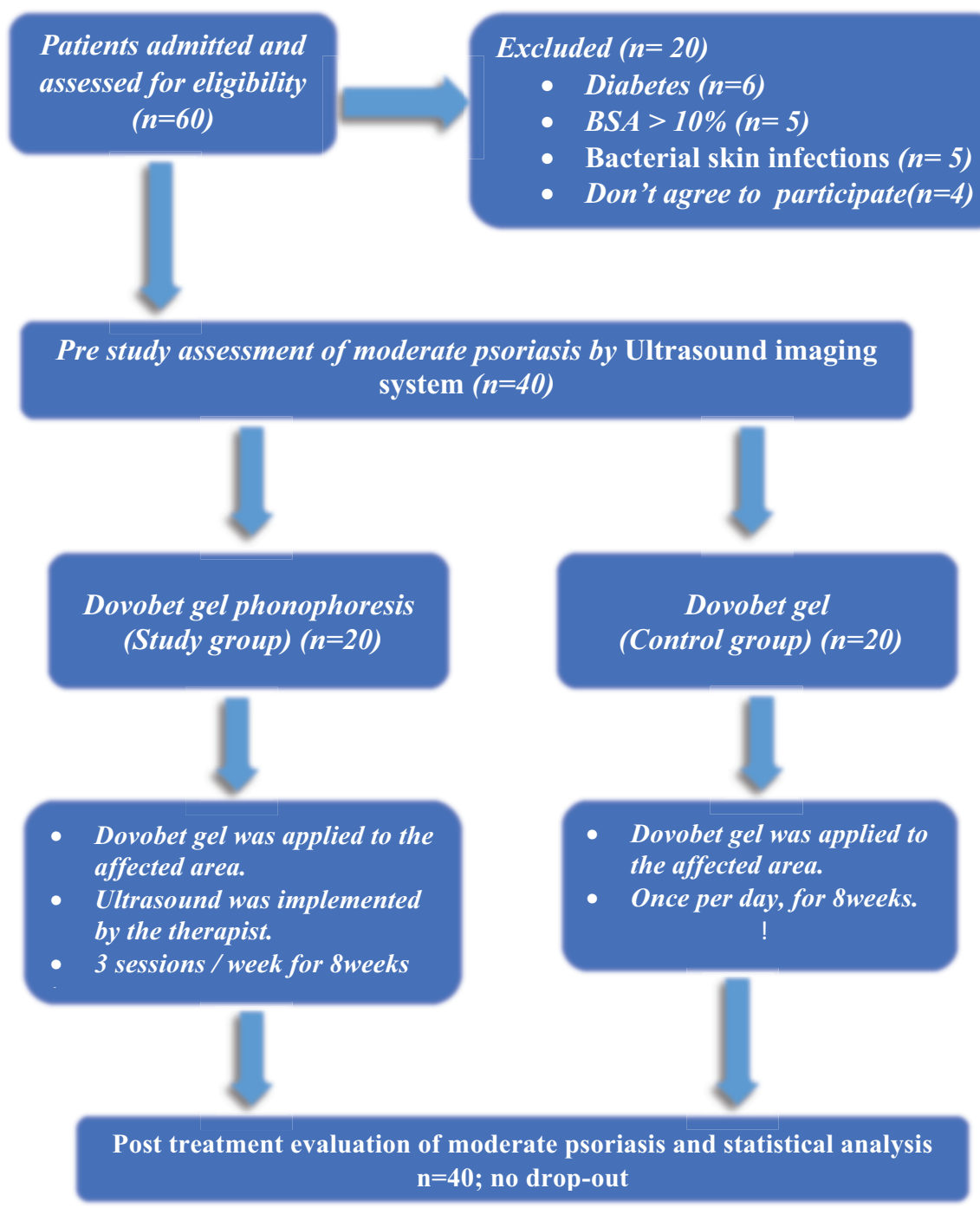

Participant flow chart. Dovobet gel phonophoresis, Dovobet gel only.

thickness between the study and control groups. Paired $t$-test was conducted for comparison between pretreatment and post-treatment values in each group. The level of significance for all statistical tests was set at $P$ value less than 0.05 .

\section{Results}

A diagram of the patients' randomization in the study is shown in Fig. 1. A total of 60 patients were initially screened. After the screening process, 40 patients were found to be eligible to participate in the study. In total, 40 (100\%) participants completed the treatment program.

\section{Demographic data of patients}

Table 1 showed the mean $\pm \mathrm{SD}$ age of the study and control groups. There was no significant difference between both groups in the mean age $(P=0.96)$.
Table 1 Comparison of patient characteristics between study and control groups

\begin{tabular}{lccccc}
\hline & $\begin{array}{c}\text { Study } \\
\text { group }\end{array}$ & $\begin{array}{c}\text { Control } \\
\text { group }\end{array}$ & MD & $\begin{array}{c}t \\
\text { value }\end{array}$ & $\begin{array}{c}P \\
\text { value }\end{array}$ \\
\hline Age $($ mean \pm SD) & 32.75 & 32.85 & -0.1 & -0.05 & 0.96 \\
(years) & \pm 6.29 & \pm 6.36 & & & $($ NS) \\
Males/females & $9 / 11$ & $10 / 10$ & - & $\begin{array}{c}0.1 \\
\left(\chi^{2}\right)\end{array}$ & $\begin{array}{c}0.75 \\
(\mathrm{NS})\end{array}$ \\
\hline
\end{tabular}

Moreover, there was no significant difference in sex distribution between groups $(P=0.75)$.

\section{Effect of treatment on skin thickness \\ Within-group comparison}

There was a significant decrease in skin thickness after treatment in the study and control groups compared with that before treatment $(P>0.001)$ (Table 2 and Figs 2 and 3). The percent decrease in skin thickness in the study and control groups was 69.23 and $38.96 \%$, respectively. 


\section{Between-group comparison}

There was no significant difference in skin thickness between both groups before treatment $(P>0.05)$. Comparison between the study and control groups after treatment revealed a significant decrease in skin thickness of the study group compared with that of the control group $(P>0.001)$.

\section{Discussion}

This study was designed to investigate the therapeutic benefits of calcipotriol plus betamethasone dipropionate gel (Dovobet gel) phonophoresis (study group) as opposed to Dovobet gel only (control group) in the treatment of psoriasis. This study was significant for two reasons. First, the study was conducted for determination of the effectiveness of topical agent, calcipotriol plus betamethasone dipropionate gel, by measuring the decrease in the skin thickness. Second,

Table 2 Mean skin thickness before treatment and after treatment in the study and control groups

\begin{tabular}{lccccc}
\hline $\begin{array}{l}\text { Skin } \\
\text { thickness } \\
(\mathrm{cm})\end{array}$ & $\begin{array}{c}\text { Study } \\
\text { group } \\
(n=20)\end{array}$ & $\begin{array}{c}\text { Control } \\
\text { group } \\
(n=20)\end{array}$ & MD & $\begin{array}{c}t \\
\text { value }\end{array}$ & $P$ value \\
\hline $\begin{array}{l}\text { Before } \\
\text { treatment }\end{array}$ & $0.78 \pm 0.09$ & $0.77 \pm 0.08$ & 0.01 & 0.38 & $0.7(\mathrm{NS})$ \\
$\begin{array}{l}\text { After } \\
\text { treatment }\end{array}$ & $0.24 \pm 0.07$ & $0.47 \pm 0.07$ & -0.23 & -9.66 & 0.001 \\
MD & 0.54 & 0.3 & & & $(\mathrm{~S})$ \\
$\begin{array}{l}\text { Percentage } \\
\text { of change }\end{array}$ & 69.23 & 38.96 & & & \\
$t$ value & 75.98 & 28.33 & & & \\
$P$ & $0.001(\mathrm{~S})$ & $0.001(\mathrm{~S})$ & & & \\
\hline
\end{tabular}

S, significant. the study was conducted to determine the effectiveness of ultrasound and topical agent on plaque psoriasis, not only regarding the relief of pruritus and erythema only but also regarding the effect of reducing the thickness of the skin.

Results of this study revealed that there was no significant difference in skin thickness between both groups before treatment $(P>0.05)$, whereas there was a significant decrease in skin thickness after treatment in study group and control group compared with that before treatment $(P<0.001)$. The percent decrease in skin thickness in the study group and control group was 69.23 and 38.96\%, respectively, and there was a significant decrease in skin thickness of the study group compared with that of the control group $(P<0.001)$.

Results of this study are supported by those of Menter et al. [10], where combined medication showed fundamentally more improvement $(27.2 \%$ of patients) compared with the gel vehicle (0.0\%), calcipotriol (11.4\%), or betamethasone dipropionate (16.9\%) monotherapy in the gel. This combination of calcipotriene/betamethasone dipropionate was again most effective in controlling disease in contrast with all other treatment groups, especially the vehicle control.

This results can be explained by $\mathrm{Ma}$ et al. [20] who reported on the once-daily calcipotriol/betamethasone with once-daily placebo versus twice-daily calcipotriol monotherapy over 12 weeks. Patients utilizing the calcipotriene/betamethasone dipropionate still had a

Figure 2

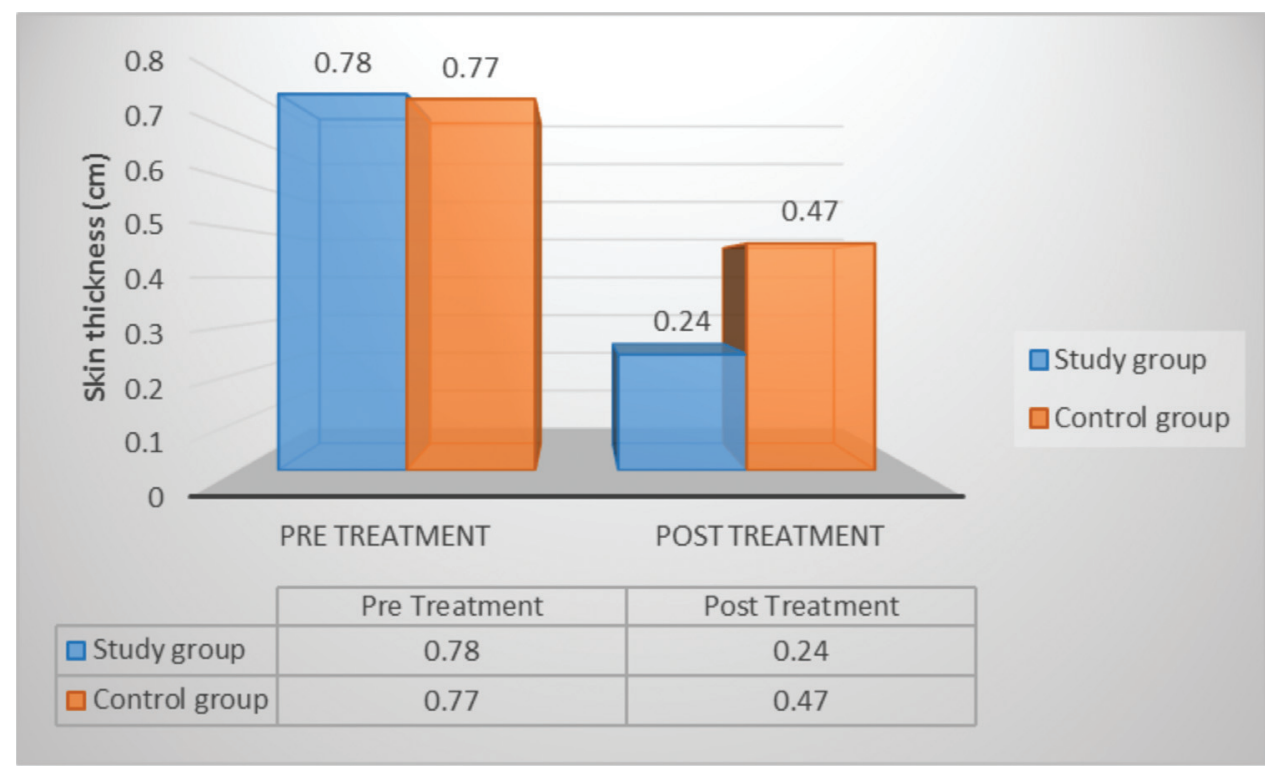

Mean skin thickness before treatment and after treatment of the study and control groups. 


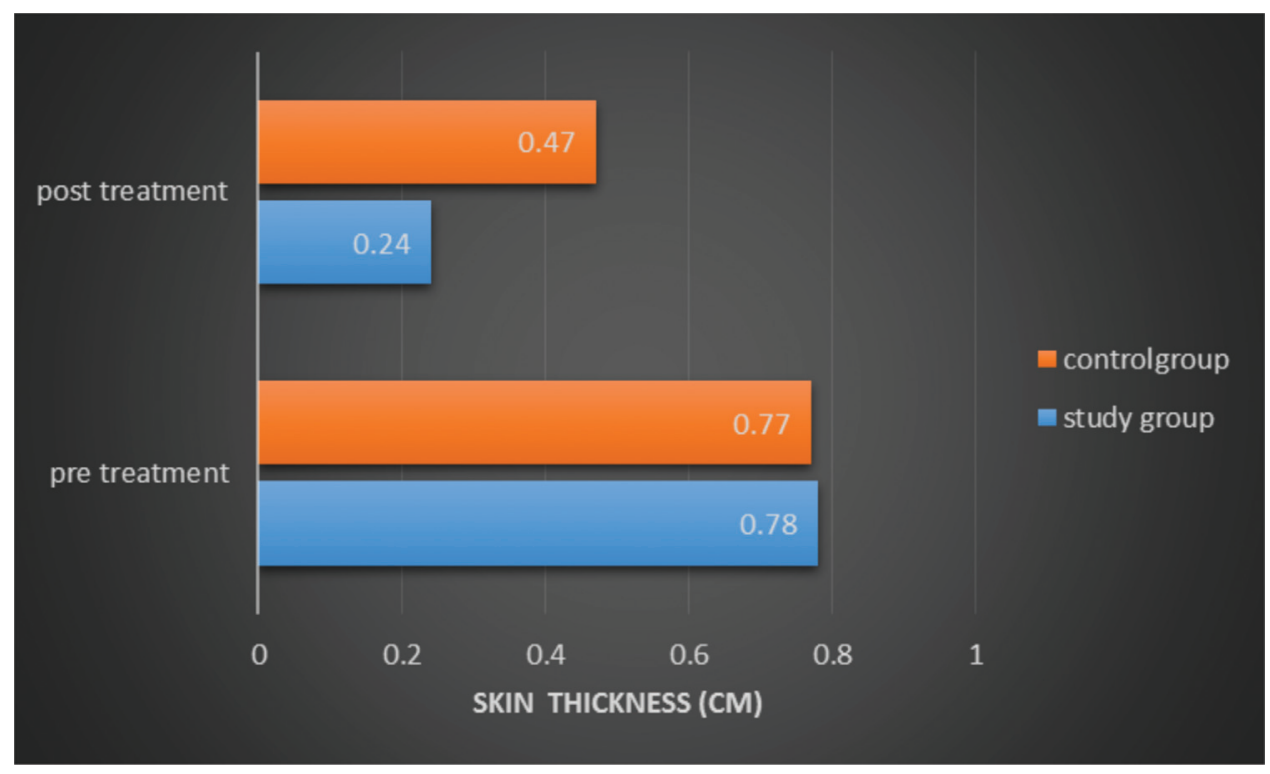

Visual bar chart determines mean skin thickness before treatment and after treatment in the study and control groups.

more prominent decrease in PASI, the area percentage of lesions, and visual analogue scale than those in the calcipotriol monotherapy group. Moreover, Huang et al. [21] showed that daily utilization of the combined for a more extended period brought about the greatest improvement. Another examination $(n=320)$, published in 2009, affirmed that after weeks 1,2 , and 4 , patients using the calcipotriene/ betamethasone dipropionate had a more significant decrease in PASI, the area percentage of lesions, and visual analogue scale than those in the calcipotriol monotherapy. Following a month, there was a $75 \%$ decline in PASI in $73.03 \%$ of patients using combination contrasted with $48.32 \%$ of patients utilizing calcipotriol monotherapy. In addition, this result can be explained by Saraceno et al. [22] who demonstrated that there was a measurably huge difference in the PASI values between treatment groups after just a week of treatment. An additional examination $(n=96)$ also assessed the utilization of the combined topical versus calcipotriol alone. Although both groups showed improvement, the combination ointment had greater adequacy and produced results in less time than could be maintained with calcipotriol alone. This agreed with Kragballe et al. [23] who detailed in a 2004 study $(n=972)$ of treatment utilizing calcipotriol/betamethasone dipropionate versus calcipotriol that the group utilizing the combined topical again had a more prominent change in PASI than patients utilizing calcipotriol either once or twice daily. This was also consisted with Papp et al. [24] who confirmed that the combination medication was more viable in diminishing PASI (73.2\%) than betamethasone dipropionate (63.1\%), calcipotriene (48.8\%), and vehicle $(28.8 \%)$ alone, utilized once every day for about a month. Moreover, the result was supported by a study conducted by Douglas et al. [25] in 2002, where 1106 patients were randomized to receive calcipotriol, betamethasone dipropionate, or the combination topically for one month. Following the treatment, the group receiving the combination treatment showed the largest decrease in PASI $(-74.4 \%)$, in contrast with the betamethasone $(-61.3 \%)$ and calcipotriol (-55.3\%) groups. There was also a statistically significant difference in PASI values between treatment groups after only 1 week of treatment.

This is agreed by Clark et al. [32] who demonstrated that the low-frequency ultrasonic debridement and cleaning might be helpful in the treatment of chronic plaque psoriasis. The mechanism of action is unclear but might be identified with the stable cavitation impacts actuated by ultrasonic energy. Low-frequency ultrasound gives viable skin debridement and cleaning in a protected and relaxing environment. Other possible mechanisms include hyperthermia, stress reduction, antiproliferative effects, and immunologic stimulation. Nevertheless, low-frequency ultrasound may demonstrate a helpful adjunct in psoriasis treatment.

The combination of vitamin $\mathrm{D}_{3}$ analogues and potent corticosteroids with low-frequency ultrasonic 
(phonophoresis) accelerates clinical response and increases efficacy. There is evidence for both shortterm and long-term efficacy and safety. The synergistic effects and the complementary mechanisms of action lead to an increased tolerability and eventually to a better adherence of the patients, so this study supports the use of Dovobet gel phonophoresis as an alternative and conjoint therapy to manage plaque psoriasis. Moreover, this result of the study is applicable to use in outclinic and for generalized population.

This study has some limitations: the small sample size and short duration of the treatment period. The findings of the study may be limited also by the lack of follow-up of psoriasis among all groups for several months after treatment program to evaluate the longlasting effect. More extensive studies assigning the efficacy of calcipotriol/betamethasone dipropionate phonophoresis on plaque psoriasis are needed. Further studies should be undertaken to use the large sample. Further studies should be carried on patients with another type of psoriasis. Further investigations are needed to compare between different frequencies and different intensities of ultrasound to detect the most beneficial frequency and intensity that can be used for drug delivery for treatment of psoriasis.

\section{Conclusion}

Calcipotriol/betamethasone dipropionate phonophoresis in a fixed combination is an effective and well-tolerated medication in mild-to-moderate psoriasis of body and scalp, and is in addition, an evidence-based treatment modality.

\section{Financial support and sponsorship \\ Nil.}

\section{Conflicts of interest}

There are no conflicts of interest.

\section{References}

1 Deng $\mathrm{Y}$, Chang C, Lu Q. The inflammatory response in psoriasis: a comprehensive review. Clin Rev Allergy Immunol 2016; 50:377-389.

2 Lønnberg AS, Skov L, Skytthe A, Kyvik KO, Pedersen OB, Thomsen SF. Heritability of psoriasis in a large twin sample. $\mathrm{Br} \mathrm{J}$ Dermatol 2013; 169:412-416.

3 Michalek IM, Loring B, John SM. A systematic review of the worldwide epidemiology of psoriasis. J Eur Acad Dermatol Venereol 2017; 31:205-212.

4 Takeshita J, Grewal S, Langan SM, Mehta NN, Ogdie A, Van Voorhees AS, Gelfand JM. Psoriasis and comorbid diseases epidemiology. J Am Acad Dermatol 2017; 76:377-390.

5 Nestle FO, Kaplan DH, Barker J. Psoriasis. N Engl J Med 2009; 361:496-509.

6 Naldi L, Mercuri SR. Smoking and psoriasis from epidemiology to pathomechanisms. J Invest Dermatol 2009; 129:2741-2743.
7 Menter A, Korman NJ, Elmets CA, Feldman SR, Gelfand JM, Gordon KB, et al. Guidelines of care for the management of psoriasis and psoriatic arthritis. Section 3. Guidelines of care for the management and treatment of psoriasis with topical therapies. J Am Acad Dermatol 2009; 60:643-659.

8 Vena GA, Galluccio A, Pezza M, Vestita M, Cassano N. Combined treatment with low-dose cyclosporine and calcipotriol/betamethasone dipropionate ointment for moderate-to-severe plaque psoriasis: a randomized controlled open-label study. J Dermatolog Treat 2012; 23:255-260.

9 National Institute for Health and Care Excellence (NICE). Clinical Guideline, 153. The assessment and management of psoriasis. October 2012. Available at: http://www.nice.org.uk/guidance/cg153. Accessed September 2017.

10 Menter A, Gold LS, Bukhalo M, Grekin S, Kempers S, Boyce BM, et al. Calcipotriene plus betamethasone dipropionate topical suspension for the treatment of mild to moderate psoriasis vulgaris on the body: a randomized, double-blind, vehicle-controlled trial. J Drugs Dermatol 2013; 12:92-98.

11 Langley RG, Gupta A, Papp K, Wexler D, Østerdal ML, Curčić D. Calcipotriol plus betamethasone dipropionate gel compared with tacalcitol ointment and the gel vehicle alone in patients with psoriasis vulgaris: a randomized, controlled clinical trial. Dermatology 2011; 222:148-156.

12 Segaert S, Røpke M. The biological rationale for use of vitamin D analogues in combination with corticosteroids for the topical treatment of plaque psoriasis. J Drugs Dermatol 2013; 12:129-137.

13 Van de Kerkhof PC, Hoffmann V, Anstey A, Barnes L, Bolduc C, Reich K, et al. A new scalp formulation of calcipotriol plus betamethasone dipropionate compared with each of its active ingredients in the same vehicle for the treatment of scalp psoriasis: a randomized, double-blind, controlled trial. $\mathrm{Br}$ J Dermatol 2009; 160:170-176.

14 Sticherling M, Eicke C, Anger T. Practicability of combined treatment with calcipotriol/betamethasone gel (Daivobet gel) and improvement of the quality of life in patients with psoriasis. J Dtsch Dermatol Ges 2013; 11:420-427.

15 Lee KL, Zhou Y. Quantitative evaluation of sonophoresis efficiency and its dependence on sonication parameters and particle size. J Ultrasound Med 2015; 34:519-526.

16 Park D, Park H, Seo J, Lee S. Sonophoresis in transdermal drug delivery. Ultrasonics 2014; 54:56-65.

17 Akinbo S, Owoeye O, Adesegun S. Comparison of the therapeutic efficacy of diclofenac sodium and methyl salicylate phonophoresis in the management of knee osteoarthritis. Arch Rheumatol 2011; 26: 111-119.

18 Goraj-Szczypiorowska B, Zajac L, Skalska-Izdebska R. Evaluation of factors influencing the quality and efficacy of ultrasound and phonophoresis treatment. Ortop Traumatol Rehabil 2007; 9:449-458.

19 Srbely S. Ultrasound in the management of osteoarthritis: part I: a review of the current literature. J Can Chiropr Assoc 2008; 52:30-37.

20 Li M, Lan H, Huang Qiong YQ. Efficacy, safety and quality of life of topical calcipotriol betamethasone dipropionate in the treatment of psoriasis vulgaris. Chinese J New Drugs Clin Remed 2013. Available from: http:// en.cnki.com.cn/Article_en/CJFDTOTAL-XYYL201306009.htm. [Accessed August 21, 2017].

21 Huang L, Li M, Huang Q, Yang QP, Zeng ZZ, Zhu XJ, et al. Calcipotriol betamethasone ointment in the treatment of psoriasis vulgaris: a randomized, double-blind,active-controlled, parallel group study. Chinese J Dermatol 2009; 42:691-694.

22 Saraceno R, Andreassi L, Ayala F, Bongiorno MR, Giannetti A, Lisi P, et al. Efficacy, safety and quality of life of calcipotriol/betamethasone

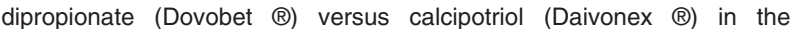
treatment of psoriasis vulgaris: a randomized, multicentre, clinical trial. J Dermatolog Treat 2007; 18:361-365.

23 Kragballe K, Noerrelund KL, Lui H, Ortonne JP, Wozel G, Uurasmaa T, et al. Efficacy of once-daily treatment regimens with calcipotriol/ betamethasone dipropionate ointment and calcipotriol ointment in psoriasis vulgaris. Br J Dermatol 2004; 150:1167-1173.

24 Papp KA, Guenther L, Boyden B, Larsen FG, Harvima RJ, Guilhou JJ, et al. Early onset of action and efficacy of a combination of calcipotriene and betamethasone dipropionate in the treatment of psoriasis. J Am Acad Dermatol 2003; 48: 48-54.

25 Douglas WS, Poulin Y, Decroix J, Ortonne JP, Mrowietz U, Gulliver W, et al. A new calcipotriol/betamethasone formulation with rapid onset of action was superior to monotherapy with betamethasone dipropionate or calcipotriol in psoriasis vulgaris. Acta Derm Venereol. 2002; 82:131-135. 
26 Welkowitz J, Ewen RB, Cohen J. Introductory statistics for the behavioura sciences. 3rd ed. San Diego, CA: Harcourt Brace Jovanovich; 1982.

27 Doig GS, Simpson F. Randomization and allocation concealment: a practical guide for researchers. J Crit Care 2005; 20:187-193.

28 Leading a Digital Industrial Era - 2016 Annual Report' (PDF). General Electric Company. https://www.ge.com/ar2016/assets/pdf/ GE_AR16_Integrated_Summary_Report.pdf. Retrieved 2017-06-15.

29 El-Zawahry MB, Abdel El-Hameed El-Cheweikh HM, Abd-El-Rahman Ramadan S, Ahmed Bassiouny D, Mohamed Fawzy M. Ultrasound biomicroscopy in the diagnosis of skin diseases. Eur J Dermatol 2007; 17:469-475.

30 Vogt M, Helmut Ermert H. Development and evaluation of a high-frequency ultrasound-based system for in vivo strain imaging of the skin. IEEE Trans Ultrason Ferroelectr Freq Control 2005; 52:375-385.

31 Loyd V, Allen J. Compounding for phonophoresis. Secundum Artem 2005 1:10-17.

32 Clark AR, Feldman SR, Jorizzo JL. Ultrasonic therapy for psoriasis. J Eur Acad Dermatol Venereol 1995; 4:58-61. 